\title{
Macrophage Activation Syndrome in Children: Diagnosis and Management
}

\author{
Narendra Kumar Bagri, ${ }^{1}$ Latika Gupta, ${ }^{2}$ Ethan S Sen, ${ }^{3}$ AV Ramanan ${ }^{4}$ \\ From ${ }^{1}$ Division of Pediatric Rheumatology, Department of Pediatrics, AIIMS, New Delhi; India; ${ }^{2}$ Department of Clinical Immunology \\ and Rheumatology, Sanjay Gandhi Postgraduate Institute of Medical Sciences, Lucknow, Uttar Pradesh, India; ${ }^{3}$ Department of \\ Pediatric Rheumatology, Great North Children's Hospital, and Faculty of Medical Sciences, Newcastle University, Newcastle Upon \\ Tyne, UK; ${ }^{4}$ Department of Pediatric Rheumatology, University Hospitals Bristol NHS Foundation Trust \& Translational Health \\ Sciences, University of Bristol, Bristol, UK. \\ Correspondence to: Dr Narendra Kumar Bagri, Associate Professor, Division of Pediatric Rheumatology, Department of Pediatrics, \\ All India Institute of Medical Sciences, New Delhi 110027,India.drnarendrabagri@yahoo.co.in
}

Macrophage activation syndrome is a severe yet under-recognized complication encountered in pediatric rheumatology. It manifests as secondary hemophagocytic lymphohistiocytosis leading to a hyper-inflammatory state resulting from an underlying cytokine storm. If unchecked, it may lead to multiorgan failure and mortality. Early diagnosis and timely initiation of specific therapy is pivotal for a successful outcome. This review outlines the key clinical and laboratory features and management of macrophage activation syndrome.

Keywords: Hyper-inflammation, Secondary hemophagocytic lymphohistiocytosis, Juvenile idiopathic arthritis.

Published online: March 26, 2021; PII: S097475591600308

$\mathrm{M}$ acrophage activation syndrome (MAS) is a form of secondary hemophagocytic lymphohistiocytosis (sHLH) and one of the most commonly encountered pediatric rheumatic emergencies [1]. While these terms are often used interchangeably, MAS is typically used for sHLH with an underlying rheumatic disorder. MAS is most often reported with systemic juvenile idiopathic arthritis (sJIA) [2]. Additionally, it is seen with Kawasaki disease (KD), juvenile systemic lupus erythematosus (SLE), and juvenile dermatomyositis (JDM) [3,4]. A single centre analysis of 31 children with MAS identified the under-lying disease as sJIA in $84 \%$, SLE in $13 \%$ and KD in $3 \%$ with the overall mortality being $32 \%$ [5]. MAS can compli-cate any chronic inflammatory condition at onset or during the course of the disease [6-8]. sHLH can also be triggered by a variety of other inflammatory conditions viz., underlying infection, malignancy or primary immuno-deficiencies [9]

MAS leads to fulminant manifestations in nearly $10 \%$ of children with sJIA, though up to $30 \%$ of children with sJIA may have subclinical MAS $[2,7,10]$. A retrospective study from India reported MAS affecting $1.3 \%$ of 950 children diagnosed with $\mathrm{KD}$ and coronary artery abnormalities were more frequent, affecting $41.7 \%$ of the subset with MAS [3]. Although, MAS is increasingly recognized since its early reports in the 1980s, it seems that a significant proportion of children are either not diagnosed or diagnosed late. This review highlights the pathogenic mechanisms, salient clinical features and the management strategies for MAS.

\section{PATHOGENESIS}

The cytolytic dysfunction of natural killer (NK) cells and cytotoxic T-lymphocytes (CTL) is the key inciting event in MAS/primary HLH [9]. While recessive null mutations in PRF1, UNC13D, STXBP2, STX11, RAB27A, and LYST, which adversely affect granule processing in the CTL and NK cells' function, are known to be associated with primary HLH, there is increasing evidence to suggest that the susceptibility to cytokine storms in sHLH could be along a continuum of genetic deficiency, with monoallelic, biallelic and polygenic defects [11-13]. Of the various implicated genetic defects in fHLH, LYST, MUNC13-4, and $S T X B P 2$, nearly one-third were detected in subjects with sJIA, MAS on whole-exome sequencing. The described heterozygous mutations may constitute the first hit in the genetically-susceptible individual resulting in a subtle underlying impairment in the cytolytic pathways, that turns into an exaggerated immune response subsequent to a second trigger by either an unknown/self-antigen (as in juvenile SLE) or an infectious agent (viral, bacterial, or fungal). This would potentially culminate in an unrestrained activation of immune cells, and subsequent frank hyperinflammatory state. Antigen-induced $\mathrm{T}$ cell hyperstimulation in MAS/sHLH is not matched with degranulation due to the underlying cytolytic dysfunction. Such persistent activation of CTL and macrophages results in a cytokine storm; most notably involving the massive release of TNF and interleukins: IL-6, IL-1b, and IL-18 [9]. The resultant cytokine storm usually exhibits good 
correlation with serum ferritin levels. Ferritin is the storage form of iron, and is an acute phase reactant. Cytokinemediated macrophage activation in MAS results in hepatic injury and release of ferritin [14]. One of the most consistent laboratory markers of MAS/sHLH is hyper-ferritinemia. Amongst other effects, ferritin has been shown to inhibit lymphocyte division and granulocyte proliferation.

Activation of macrophages leads to hemophagocytosis, a cardinal feature of MAS that can be demonstrated histopathologically in the bone marrow, liver, and spleen. However, bone marrow examination shows HLH with positive CD163 macrophages in only up to $60 \%$ of cases, and this finding may not be evident during the initial stages [15]. CD163 and CD25 are markers of activated macrophages which mediate endocytosis of haptoglobinhemoglobin complexes, and the elevated levels of these surface markers, i.e., soluble interleukin-2 receptor alpha chain (sCD25) and soluble CD163 (sCD163) may be sensitive indicators of detecting MAS. Fig. 1 demonstrates the pathogenic mechanism for MAS.

The prototype animal model of sJIA MAS is the IL-6 transgenic mouse, wherein LPS induces a hyperinflammatory state akin to MAS, thus recapitulating the occurrence of infection-triggered sJIA MAS. While IL-6induced stimulation of macrophages accentuates further cytokine production (excess IL-1, IL-6 or IL-18), in vitro experiments have suggested its association with a transient NK cell dysfunction resulting in decreased degranulation i.e., low perforin and granzyme expression on stimulated NK cells in the inflamed microenvironment
[16]. The other crucial arm of inflammation is macrophage hyperfunction, with various defects translating into a systemic hyperin-flammatory state. The macrophage phenotype also changes from M1 (pro-inflammatory) to M2 (anti-inflammatory) in response to external stimuli amid the changing inflammatory milieu [9]. The underlying hyper-cytokinemia and inflammatory cascade form the basis for anti-cytokine therapies in sJIA.

Apart from sJIA, MAS can complicate other rheumatic disorders like juvenile SLE, KD and JDM. In juvenile SLE, defective clearance of self-antigens (due to defects in the dendritic cells and the complement cascade) may contribute to MAS. There is some evidence that the different cytokines may have a major role in the pathogenesis of MAS depending on the underlying rheumatic disorder: soluble TNF receptor (sTNFR)-I levels for SLE, serum IL-18 levels for JDM, and serum sTNFR-II levels for KD and sJIA [17]. A study from Japan, which measured cytokine levels in 36 patients with MAS complicating sJIA, showed a positive correlation of serum sTNFR-II/I ratio with disease activity. This correlation still appeared to exist in the patients treated with the anti-IL-6 drug tocilizumab [18]. The authors suggest that the serum sTNFR-II/I ratio may be a helpful biomarker to assist diagnosis of MAS in sJIA patients receiving tocilizumab.

\section{CLINICAL AND LABORATORY FEATURES}

Timely recognition of MAS is of utmost importance for suitable management and successful outcome. Ahigh degree of suspicion is warranted, and it should be considered in any febrile child with underlying rheumatic disease. The

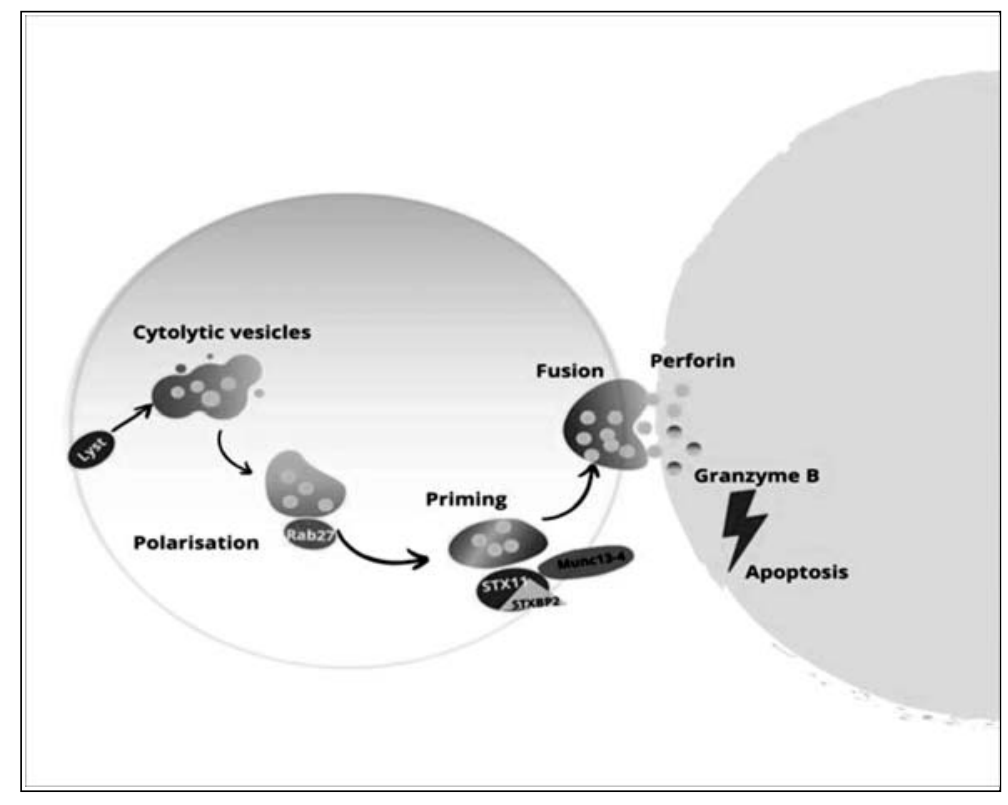

Fig. 1 Immune pathways implicated in macrophage activation syndrome. 
persistent high-grade fever, especially in those who are not on any long term immunosuppression, is one of the most important features of MAS. The typical fever in sJIA changes from an intermittent pattern to a non-remitting fever in MAS, and this change in the pattern of fever may be an early clue to differentiate MAS from the underlying disease flare. However, sometimes children with ongoing immunosuppression (steroids) may not mount the typical high persistent fever but have other clinical and laboratory features suggestive of MAS. Absence of fever has also been observed in children on biologicals [15], and it seems that fever may not be an absolute finding in all cases of MAS.

The underlying cytokine storm in MAS results in multiorgan involvement and can have varied clinical features. Many patients will have hepatosplenomegaly and lymphadenopathy. Hemorrhagic manifestations secondary to thrombocytopenia, coagulopathy and liver dysfunction include petechial spots, mucosal bleeding, severe gastrointestinal bleeding, and disseminated intravascular coagulation. Central nervous system (CNS) involvement in the form of drowsiness, headache, seizures and altered behavior; from mild confusional state to frank coma, has been reported in 30-35\% patients; and often heralds a worse prognosis. Unless promptly recognized, MAS can lead to multiorgan involvement, including cardiac, pulmonary, and renal failure. The reported mortality of MAS varies from 10-35\%; however, early recognition and prompt treatment can improve survival rates. Differentiating the underlying disease flare and MAS is often challenging Table I summarizes the major differences.

MAS in SLE: MAS is most commonly described with sJIA; however, it is increasingly being recognized with other rheumatic disorders, particularly SLE. Based on international cohort data, patients with oral/nasal ulcers, arthritis, serositis, renal, CNS or hematologic involvement at diagnosis of SLE are more likely to develop MAS [19]. Borgia, et al. [8] have reported that $9 \%$ of children with SLE had MAS, and the majority ( $68 \%$ ) had features of MAS at the disease onset. The diagnosis of MAS in SLE is often more challenging because of the overlapping features like fever, organomegaly and cytopenias in both disease flare and MAS. However, lymphadenopathy and cytopenias are more frequently observed in MAS as compared to active SLE. While comparing the clinico-laboratory differences between MAS and active SLE without MAS, all clinical features of MAS except fever have better specificity than sensitivity. In contrast, fever is the most sensitive with low specificity. Hyperferritinemia, hypertriglyceridemia, elevated lactate dehydrogenase and hypofibrinogenemia are known to have the best sensitivity and specificity in differentiating MAS from active lupus [15]. Akin to sJIA, MAS in SLE can be life-threatening, with mortality ranging from $5-11 \%[8,15]$ and therefore needs a high index of suspicion for timely diagnosis of MAS in this population.

\section{CLASSIFICATION CRITERIA AND DIAGNOSIS}

The diagnosis of MAS is often challenging, especially

Table I Differentiating Features Between Macrophage Activation Syndrome and Disease Flare

\begin{tabular}{|c|c|c|c|}
\hline \multirow[t]{2}{*}{ Clinical and laboratory parameter } & \multirow[t]{2}{*}{ Macrophage activation syndrome } & \multicolumn{2}{|c|}{ Disease flare } \\
\hline & & SLE & SJIA \\
\hline Fever & +++ & ++ & ++ \\
\hline Hepato-splenomegaly & + & \pm & \pm \\
\hline Hemorrhages & ++ & - & - \\
\hline CNS dysfunction & ++ & \pm & - \\
\hline Hemoglobin & Low & Normal to low & Normal \\
\hline Platelet count & Low & Normal to low & Highly elevated \\
\hline Erythrocyte sedimentation rate & Normal to low & Normal to elevated & Normal to elevated \\
\hline C-reactive protein & Elevated & Normal to elevated & Normal to elevated \\
\hline Aspartate aminotransferase & Elevated & Elevated & Normal \\
\hline Alanine aminotransferase & Elevated & Normal & Normal \\
\hline Lactate dehydrogenase & Elevated & Normal & Normal \\
\hline Ferritin $^{a}$ & Elevated & Normal & Normal \\
\hline Fibrinogen & Low & Normal & Normal \\
\hline Triglycerides & Elevated & Normal & Normal \\
\hline
\end{tabular}

${ }^{a}$ Hyperferritinemia has the best sensitivity and specificity, followed by increased lactate dehydrogenase level, hypertriglyceridemia, and hypofibrinogenemia for diagnosis of MAS. SLE: systemic lupus erythematosus, SJIA: systemic onset juvenile idiopathic arthritis. 
Box I Pediatric Rheumatology International Trials Organisation Macrophage Activation Syndrome Classification Criteria (2016) [2]

A febrile child with known or suspected SJIA is classified as having MAS if the serum ferritin $>684 \mathrm{ng} / \mathrm{mL}$ and $\geq 2$ of the following:

- Platelets $\leq 181 \times 10^{9} / \mathrm{L}$

- Aspartate aminotransferase (AST) $>48 \mathrm{U} / \mathrm{L}$

- Triglycerides $>156 \mathrm{mg} / \mathrm{dL}$

- Fibrinogen $\leq 360 \mathrm{mg} / \mathrm{dL}$

during the early phase of the disease. The HLH-2004 criteria, originally designed for enrolment in clinical treatment trial, are often used for classification of MAS but lack sensitivity, especially for early detection of MAS. In addition, some of the features described in the HLH-2004 criteria overlap with features observed in active rheumatic diseases in the absence of MAS. Some of these challenges have been addressed in the proposed 2016 MAS in sJIA classification criteria by the Pediatric Rheumatology International Trials Organisation (PRINTO) (Box I), which have a sensitivity of $73 \%$ and specificity of $99 \%$ [2]. Although originally described for children with sJIA, these criteria have also been found to be useful for other rheumatic conditions like SLE and KD. Recently, these criteria have also been used to classify MAS associated with pediatric multisystem inflammatory syndrome temporally related with COVID-19 (PIMS-TS) [20].

The various laboratory parameters including falling total white cell count, low platelet counts, low fibrinogen and low ESR, suggest the diagnosis of MAS. It is difficult to clinch the diagnosis of MAS using a single clinical or laboratory parameter. Kostik, et al. [21] proposed a combination of more than three of the following laboratory parameters to predict an early diagnosis of MAS in sJIA reliably: declining platelet $\left(\leq 211 \times 10^{9} / \mathrm{L}\right)$ and white blood cell counts $\left(\leq 9.9 \times 10^{9} / \mathrm{L}\right)$; decreased albumin $(\leq 2.9 \mathrm{~g} / \mathrm{dL})$ and fibrinogen $(\leq 1.8 \mathrm{~g} / \mathrm{L})$; elevated ferritin $(>400 \mu \mathrm{g} / \mathrm{L})$, aspartate aminotransferase $(>59.7 \mathrm{U} / \mathrm{L})$ and lactate dehydrogenase $(>882 \mathrm{U} / \mathrm{L})$; and proteinuria.

More recently in 2019, the novel MAS/sJIA(MS) score was developed in an attempt to improve discrimination between active sJIA and MAS [21]. An international cohort of several hundred patients with sJIA, with and without MAS, were analyzed to identify clinical and laboratory parameters which distinguished the conditions. The MS score includes seven variables: central nervous system dysfunction, hemorrhagic manifestations, active arthritis, platelet count, fibrinogen, lactate dehydrogenase and ferritin. The total score ranges between -8.4 to 41.8 with a value $\geq-2.1$ showing the best performance. Although the authors reported a good performance in a validation cohort, a separate study compared the MS score with the Hscore for identification of MAS in 71 patients with sJIA and found that the H-score performed slightly better [23].

An additional measure to help recognition of MAS in the context of sJIA, which is simpler than the MS score or $\mathrm{H}$-score, is the ferritin to ESR ratio. A study using data from a large international cohort found that a ferritin/ESR ratio $>21.5$ has a sensitivity and specificity of $82 \%$ and $78 \%$, respectively for diagnosing sJIA-MAS versus active sJIA without MAS [24]. In light of the challenges faced while applying the available criteria in clinical practice, it is crucial to meticulously observe for evolving clinical features and trends of laboratory variables in order not to miss a diagnosis of MAS.

Hyperferritinemia forms part of the HLH-2004 and the 2016 MAS in sJIA criteria with thresholds of $500 \mathrm{ng} / \mathrm{mL}$ and $684 \mathrm{ng} / \mathrm{mL}$, respectively. In clinical practice, in patients diagnosed with MAS, ferritin is usually $>1000 \mathrm{ng} / \mathrm{mL}$ and frequently $>10,000 \mathrm{ng} / \mathrm{mL}$. In a systematic review by Sarangi, et al. [15], the median ferritin in children with MAS, sepsis and familial HLH was $37680 \mathrm{ng} / \mathrm{mL}, 8775 \mathrm{ng} / \mathrm{mL}$ and $3234 \mathrm{ng} / \mathrm{mL}$, respectively. One study, which analyzed all children admitted to Texas Children's Hospital over two years with ferritin $>500 \mathrm{ng} / \mathrm{mL}$, found that a ferritin $>10000$ $\mathrm{ng} / \mathrm{mL}$ was $90 \%$ sensitive and $96 \%$ specific for HLH [26]. A retrospective analysis over three years of children with ferritin $>10000 \mathrm{ng} / \mathrm{mL}$ at 11 UK centres identified 153 patients, but HLH/MAS was not entertained as a diagnostic possibility in $23.2 \%$ of these children [27]. This

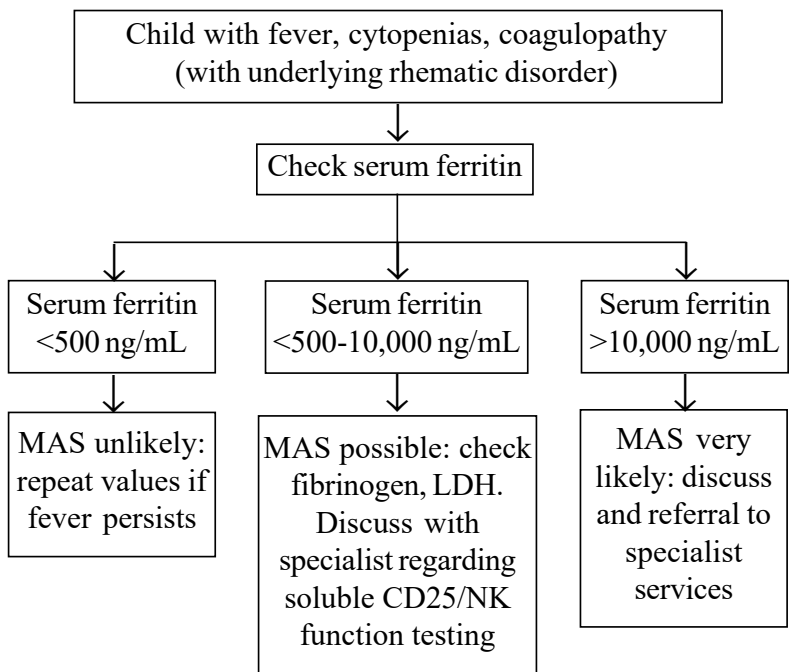

Fig. 2 Guide to early diagnosis and referral for macrophage activation syndrome (adapted from: Sen ES, Steward CG, Ramanan AV. Diagnosing haemophagocytic syndrome. Arch Dis Child. 2017;102:279-84 [287). 
highlights the importance of improving education and awareness of HLH/MAS amongst all clinicians caring for children. We recommend that a child with fever, cytopenias and/or coagulopathy with ferritin $>10000 \mathrm{ng} / \mathrm{mL}$ be referred to specialist services for evaluation and management of probable MAS/sHLH (Fig. 2) [28].

\section{TREATMENT}

Timely diagnosis and intervention are pivotal for dampening the underlying cytokine storm and curtailing the morbidity and mortality associated with MAS. The treatment is aimed at mitigating the underlying cytokine storm using immunosuppression and controlling the inciting trigger. In clinical practice, ascertaining the underlying infectious trigger is often not possible. Empirical antibiotics and/or antifungals are instituted in parallel with the immunosuppressive therapy to cover the probable infectious trigger. The treatment of MAS secondary to sJIA is based on the evidence generated from case series, and the same protocol is usually extrapolated for management of MAS secondary to other rheumatic disorders [29-31].

Intravenous methylprednisolone (IVMP) pulse therapy, $30 \mathrm{mg} / \mathrm{kg} /$ day (maximum $1 \mathrm{~g}$ ) for 3 to 5 days, followed by oral prednisolone is the most widely used first-line therapy $[31,32]$. The oral steroids are initiated at a dose of $2 \mathrm{mg} / \mathrm{kg} /$ day and gradually tapered while monitoring the clinical and laboratory parameters. They are usually continued for a few weeks until normalisation of hematological abnormalities. Subsequent doses of steroids are titrated as per the need of the underlying disease. There are a few reports of replacing IVMP with dexamethasone palmitate (dose varying from 7.5 to $10 \mathrm{mg} /$ day) in refractory cases or CNS involvement in MAS [33].

The calcineurin inhibitor cyclosporine A has shown efficacy in other histiocytic disorders, and this has led to its use in MAS. Addition of cyclosporine with steroids in the management of MAS results in rapid control of the underlying cytokine storm, in addition to limiting the excessive use of corticosteroids. However, in the acute setting of patients with multiorgan dysfunction, cyclosporine should be used with caution due to its neurotoxic and nephrotoxic side effects. The optimal duration of cyclosporine in MAS is unclear, and it has been used for a variable duration from 3 months to 2 years [34]. It should be continued until the normalization of all laboratory parameters, followed by tapering to prevent relapses. We usually consider oral cyclosporine in a dose of $2-7 \mathrm{mg} / \mathrm{kg} /$ day for a period of 3-6 months.

Intravenous immunoglobulin (IVIG) has also been used in the treatment of MAS, and it may be considered as adjunctive therapy to IVMP $[35,36]$. Given the relative safety and favorable adverse effect profile, it seems prudent to use IVIG as an adjunct to IVMP, especially in Indian settings where underlying infection always remains a possibility. We usually consider using IVIG as initial therapy when the clinical suspicion of concomitant infection is high.

Cytokine-specific therapy using biological agents like anti-IL-1 (anakinra) is increasingly used in the treatment of MAS in sJIA; with improved benefit with an early institution [37]. The use of anakinra is usually considered at an early stage if there is no response to IVMP [30]. Although a common starting dose of anakinra is $2 \mathrm{mg} / \mathrm{kg} /$ day, it may be escalated up to $8 \mathrm{mg} / \mathrm{kg} /$ day in refractory cases. In cases of shock and poor peripheral absorption, intravenous anakinra can be recommended [38]. Other anti-cytokine therapeutic options for use in MAS include IL-1 $\alpha$ and IL-18 blockers [39]. However, non-availability of these agents precludes their use in India. The utility of other biological agents like anti-IL-6 receptor monoclonal antibody tocilizumab and anti-TNF agents (etanercept) in treating MAS secondary to various

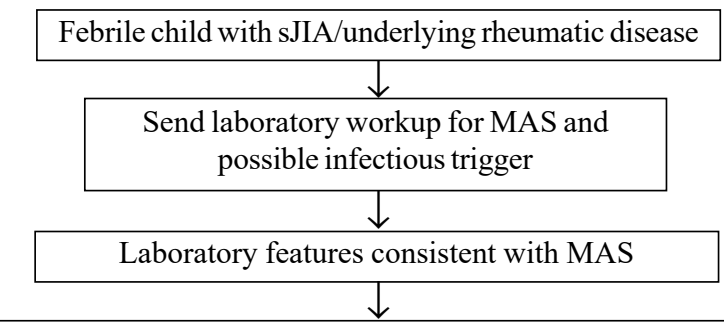

IVMP $30 \mathrm{mg} / \mathrm{kg} /$ day for 3-5 days, followed by oral prednisolone $2 \mathrm{mg} / \mathrm{kg} /$ day with gradual tapering ${ }^{b}$ $\pm \mathrm{IVIG}^{a} 1 \mathrm{gm} / \mathrm{kg}$ for 2 days may be repeated at 2 weeks (Consider anakinra, starting 1-2 mg/kg ( $\max 8 \mathrm{mg} / \mathrm{kg}$ ), wherever available) and

Cyclosporinc $2-7 \mathrm{mg} / \mathrm{kg} /$ day followed by tapering ${ }^{b}$ (3-6 months or more) and

Parallel treatment with empirical antibiotics/antifungals

Rituximab may be considered for EBV-associated MAS

2nd line options include etoposide and other agents Needs shared decision making in colaboration with hemato-oncology team

Emapaulamb; anti-INF- $\gamma$ monoclonal antibody may be a potential therapy for refractory cases

${ }^{a}$ Initiating IVIG where underlying infection cannot be ruled out seems a reasonable practical approach. In such scenarios IVMP can be given after $48 \mathrm{hr}$ of IVIG and antibiotic coverage; ${ }^{b}$ gradual tapering based on clinical and hematological parameters.

Fig. 3 Management strategy for macrophage activation syndrome (MAS). 
rheumatic disorders remains unclear. The children with sJIA on tocilizumab may remain afebrile with lower cell counts and ferritin levels while they develop MAS, compared to those developing MAS without tocilizumab therapy [40]. As discussed previously, the serum sTNFR-II/I ratio may be a helpful biomarker to identify the onset of MAS in these patients on tocilizumab treatment [18]. Rituximab, a monoclonal antibody to $\mathrm{CD} 20$, may be considered in EBVtriggered MAS [41]. A dose of $375 \mathrm{mg} / \mathrm{m}^{2}$ weekly for four weeks has shown successful results in this setting.

MAS refractory to initial IVMP and cyclosporine is often challenging to manage. In refractory cases, additional use of etoposide may be considered [42]. However, this may be complicated with fatal myelosuppression and opportunistic infections. There are anecdotal reports of successful outcomes with the use of cyclophosphamide in refractory MAS $[42,43]$. Recently, emapalumab, a fully human IgG1 anti-INF- $\gamma$ monoclonal antibody has shown its efficacy in children with primary HLH [44]. There is preliminary data suggesting effectiveness of emapalumab in MAS associated with sJIA, although this, and its potential role in MAS secondary to other underlying rheumatic disorders, needs to be further studied [45]. Treatment of refractory MAS is often challenging and should be tailored judiciously in consultation with the hemato-oncology team. Fig. 3 summarizes the treatment approach for MAS. Hematopoietic stem cell transplantation (HSCT) is a curative option for primary/familial HLH. ten Cate, et al. [46] shared their experience of allogenic stem cell transplant carried out in a child with refractory sJIA who succumbed to MAS following this modality [46]. With the availability of more specific cytokine-targeted therapy with favorable outcome, HSCT is not advocated for treatment of refractory MAS.

\section{CONCLUSION}

Macrophage activation syndrome is one of the commonest pediatric rheumatic emergencies. It is most frequently reported with sJIA; although, it can be a complication of any underlying rheumatic disorder. Impaired cytolytic function and the resultant cytokine storm lead to laboratory and clinical manifestations, including, in some cases, fulminant multiorgan dysfunction. Timely diagnosis and judicious use of immunosuppressive agents is pivotal for a successful outcome.

Contributors: NKB, AVR: conception of idea; NKB: reviewed the literature, prepared the initial draft; LG,ES,AVR: reviewed the draft and revised it critically for important intellectual content; The final version of the manuscript have been critically revised and approved by all authors.

Funding: None; Competing interest: None stated.

\section{REFERENCES}

1. Bagri NK. Emergencies in Pediatric Rheumatology. In: P Gupta, editor. Principles of Pediatric and Neonatal Emer-gencies. 4th ed. Jaypee Publishers; 2020. p. 538-47.

2. Ravelli A, Minoia F, Davì S, et al. 2016 Classification Criteria for Macrophage Activation Syndrome Complicating Systemic Juvenile Idiopathic Arthritis: A European League Against Rheumatism/American College of Rheumatology/Paediatric Rheumatology International Trials Organisation Collabora-tive Initiative. Arthritis Rheumatol. 2016; 68: 566-76.

3. Pilania RK, Jindal AK, Johnson N, et al. Macrophage activation syndrome in children with Kawasaki disease: An experience from a tertiary care hospital in northwest India. Rheumatology (Oxford). 2020 Nov 22:keaa715.

4. Poddighe D, Dauyey K. Macrophage activation syndrome in juvenile dermatomyositis: A systematic review. Rheumatol Int. 2020;40:695-702.

5. Pal P, Bathia J, Giri PP, Roy M, Nandi A. Macrophage activation syndrome in pediatrics: 10 years data from an Indian center. Int $\mathrm{J}$ Rheum Dis. 2020 Jul 14.

6. Garcýa-Pavon S, Yamazaki-Nakashimada MA, Baez M. Kawasaki Disease Complicated With Macrophage Activation Syndrome: A Systematic Review. J Pediatr Hematol Oncol. 2017; $39: 7$.

7. Behrens EM, Beukelman T, Paessler M, Cron RQ. Occult macrophage activation syndrome in patients with systemic juvenile idiopathic arthritis. J Rheumatol. 2007;34:1133-8.

8. Borgia RE, Gerstein M, Levy DM, Silverman ED, Hiraki LT. Features, treatment, and outcomes of macrophage activation syndrome in childhood-onset systemic lupus erythematosus. Arthritis Rheumatol. 2018;70:616-24.

9. Crayne CB, Albeituni S, Nichols KE, Cron RQ. The Immunology of macrophage activation syndrome. Front Immunol. 2019; 10:119.

10. Minoia F, Davì S, Horne A, et al. Dissecting the heterogeneity of macrophage activation syndrome complicating systemic juvenile idiopathic arthritis. J Rheumatol. 2015;42:994-1001.

11. Cetica V, Sieni E, Pende D, et al. Genetic predisposition to hemophagocytic lymphohistiocytosis: Report on 500 patients from the Italian registry. J Allergy Clin Immunol. 2016; 137: 188196.e4.

12. Kaufman KM, Linghu B, Szustakowski JD, et al. Whole exome sequencing reveals overlap between macrophage activation syndrome in systemic juvenile idiopathic arthritis and familial hemophagocytic lymphohistiocytosis. Arthritis Rheumatol. 2014;66:3486-95.

13. Strippoli R, Caiello I, De Benedetti F. Reaching the Threshold: A multilayer pathogenesis of macrophage activation syndrome. $\mathrm{J}$ Rheumatol. 2013;40:761-7.

14. Wang W, Knovich MA, Coffman LG, Torti FM, Torti SV. Serum Ferritin: Past, present and future. Biochim Biophys Acta. 2010;1800:760-9.

15. Cron RQ, Davi S, Minoia F, Ravelli A. Clinical features and correct diagnosis of macrophage activation syndrome. Expert Rev Clin Immunol. 2015;11:1043-53.

16. Bracaglia C, Prencipe G, De Benedetti F. Macrophage activation syndrome: Different mechanisms leading to a one clinical syndrome. Pediatr Rheumatol. 2017;15:5.14.

17. Mizuta M, Shimizu M, Irabu H, et al. Comparison of serum cytokine profiles in macrophage activation syndrome complicating different background rheumatic diseases in children. Rheumatology. 2020;00:1-8.

18. Irabu H, Shimizu M, Kaneko S, et al. Comparison of serum biomarkers for the diagnosis of macrophage activation syndrome complicating systemic juvenile idiopathic arthritis during tocilizumab therapy. Pediatr Res. 2020;88:934-39.

19. Parodi $A$, Davì $S$, Pringe $A B$, et al. Macrophage activation syndrome in juvenile systemic lupus erythematosus: A 
multinational multicenter study of thirty-eight patients. Arthritis Rheumat. 2009;60:3388-99.

20. Verdoni L, Mazza A, Gervasoni A, et al. An outbreak of severe Kawasaki-like disease at the Italian epicentre of the SARS-CoV-2 epidemic: An observational cohort study. Lancet. 2020;395: 1771-778.

21. Kostik MM, Dubko MF, Masalova VV, et al. Identification of the best cutoff points and clinical signs specific for early recognition of macrophage activation syndrome in active systemic juvenile idiopathic arthritis. Sem Arthritis Rheumat. 2015;44:417-22.

22. Minoia F, Bovis F, Davì S, et al. Pediatric Rheumatology International Trials Organization, the Childhood Arthritis and Rheumatology Research Alliance, the Pediatric Rheumatology Collaborative Study Group and the Histiocyte Society. Development and initial validation of the MS score for diagnosis of macrophage activation syndrome in systemic juvenile idiopathic arthritis. Ann Rheum Dis. 2019;78:1357-62.

23. Sag E, Keskin A, Atalay E, et al. Performances of the "MSscore" and "HScore" in the diagnosis of macrophage activation syndrome in systemic juvenile idiopathic arthritis patients. Rheumatol Int. 2021;41:87-93.

24. Eloseily EMA, Minoia F, Crayne CB, et al. Ferritin to erythrocyte sedimentation rate ratio: simple measure to identify macrophage activation syndrome in systemic juvenile idiopathic arthritis. ACR Open Rheumatol. 2019;1: 345-49.

25. Sarangi R, Pathak M, Padhi S, Mahapatra S. Ferritin in hemophagocytic lymphohistiocytosis (HLH): current concepts and controversies. Clinica Chimica Acta. 2020; 510: 408-15.

26. Allen CE, Yu X, Kozinetz CA, McClain KL. Highly elevated ferritin levels and the diagnosis of hemophagocytic lymphohistiocytosis: Highly elevated ferritin levels and HLH. Pediatr Blood Cancer. 2008;50:1227-35.

27. Sen E, Almeida B, Moran L, et al. Fri0541 highly elevated ferritin levels are associated with haemophagocytic lymphohistiocytosis/macrophage activation syndrome - are we missing treatable diagnoses? A retrospective service evaluation of diagnosis in patients with ferritin $>10,000$ microgram/l. In: Pediatric Rheumatology [Internet]. BMJ Publishing Group Ltd and European League Against Rheumatism; 2019. p. 965.1-965.

28. Sen ES, Steward CG, Ramanan AV. Diagnosing haemophagocytic syndrome. Arch Dis Child. 2017;102:279-84.

29. Boom V, Anton J, Lahdenne P, et al. Evidence-based diagnosis and treatment of macrophage activation syndrome in systemic juvenile idiopathic arthritis. Pediatric Rheumatology. 2015: 13:55.

30. Ishii E. Hemophagocytic lymphohistiocytosis in children: Pathogenesis and treatment. Front Pediatr. 2016;4:47.

31. Carter SJ, Tattersall RS, Ramanan AV. Macrophage activation syndrome in adults: recent advances in pathophysiology, diagnosis and treatment. Rheumatology. 2019;1;58:5-17.

32. Henderson LA, Cron RQ. Macrophage activation syndrome and secondary hemophagocytic lymphohistiocytosis in childhood inflammatory disorders: diagnosis and management. Pediatr
Drugs. 2020;22:29-44.

33. Y Nakagishi, M Shimizu, K Kasai, M Miyoshi, A Yachie. Successful therapy of macrophage activation syndrome with dexamethasone palmitate. Mod Rheumatol. 2016;26:617-20.

34. Mouy R, Stephan J-L, Pillet P, et al. Efficacy of cyclosporine A in the treatment of macrophage activation syndrome in juvenile arthritis: Report of five cases. The J Pediatrics. 1996;129: $750-4$.

35. Singh S, Chandrakasan S, Ahluwalia J, et al. Macrophage activation syndrome in children with systemic onset juvenile idiopathic arthritis: Clinical experience from northwest India. Rheumatol Int. 2012;32:881-6.

36. Nandhakumar D, Loganatha A, Sivasankaran M, Sivabalan S, Munirathnam D. Hemophagocytic lymphohistiocytosis in children. Indian J Pediatr. 2020;87:526-31.

37. Eloseily EM, Weiser P, Crayne CB, et al. Benefit of anakinra in treating pediatric secondary hemophagocytic lymphohistiocytosis. Arthritis Rheumatol. 2020;72: 326-34.

38. Mehta P, Cron RQ, Hartwell J, Manson JJ, Tattersall RS. Silencing the cytokine storm: the use of intravenous anakinra in haemophagocytic lymphohistiocytosis or macrophage activation syndrome. Lancet Rheumatol. 2020;2:e358-67.

39. Crayne C, Cron RQ. Pediatric macrophage activation syndrome, recognizing the tip of the iceberg. Euro J Rheumatol. 2020 $3 ; 7: 13-20$.

40. Schulert GS, Minoia F, Bohnsack J, et al. Effect of biologic therapy on clinical and laboratory features of macrophage activation syndrome associated with systemic juvenile idiopathic arthritis. Arthritis care and research. 2018;70:409-19.

41. Balamuth NJ, Nichols KE, Paessler M, Teachey DT. Use of Rituximab in conjunction with immunosuppressive chemotherapy as a novel therapy for Epstein Barr virus-associated hemophagocytic lymphohistiocytosis: J Pediatric Hematol Oncol. 2007;29:569-73.

42. Kumakura S, Murakawa Y. Clinical Characteristics and Treatment Outcomes of Autoimmune-Associated Hemo-phagocytic Syndrome in Adults: Autoimmune-Associated Hemo-phagocytic Syndrome. Arthritis and Rheumatol. 2014; 66:2297-307.

43. Griffin G, Shenoi S, Hughes GC. Hemophagocytic lymphohistiocytosis: An update on pathogenesis, diagnosis, and therapy. Best Pract Res Clini Rheumatol. 2020;101515.

44. Locatelli F, Jordan M.B, Allen C, et al. Emapalumab in children with primary hemophagocytic lymphohistiocytosis. N Engl J Med. 2020;382:1811-22.

45. De Benedetti F, Brogan P, Bracaglia C, et al. OP0290 Emapalumab (Anti-Interferon-Gamma Monoclonal Antibody) in patients with macrophage activation syndrome (Mas) complicating systemic juvenile idiopathic arthritis (Sjia). Ann Rheumatic Dis. 2020;79:180.

46. ten Cate R, Brinkman DM, van Rossum MA, et al. Macrophage activation Syndrome after autologous stem cell transplantation for systemic juvenile idiopathic arthritis. Eur J Pediatr. 2002;161:685-86. 